tially outside the recommendations of the EMA, the G-BA, and clinical guidelines. Such audits are very rare in practice. Limits on insurer interference reflect the recognition by health insurance plans that moderation in drug spending is to be pursued by means of control over prices rather than control over physician prescribing and patient adherence. Some observers have recommended that the United States adopt an analogous model, under which manufacturers agree to accept value-based prices in exchange for insurers reducing prior-authorization requirements and cost sharing so as not to impede appropriate patient access. ${ }^{5}$

The German system for determining drug prices features collective negotiations on the part of competing health plans rather than price regulation by a single government agency. A statutory framework that creates incentives for agreement, limits price increases not justified by new evidence, and avoids heavy burdens on physicians and patients ensures that public interests are represented in private negotiations. Perhaps most important over the long run, the German structure has gained legitimacy among its principal stakeholder groups, including physicians, patient advocates, drug manufacturers, health plans, and the broader public. It remains to be seen whether the contemporary policy debate and political turmoil in the United States will also generate an economically efficient and socially accepted drug-pricing system.
Disclosure forms provided by the author are available at NEJM.org.

From the University of California, Berkeley, School of Public Health, Berkeley.

1. Berkemeier F, Whaley C, Robinson JC. Increasing divergence in drug prices between the United States and Germany after implementation of comparative effectiveness analysis and collective price negotiations. J Manag Care Spec Pharm 2019;25: 1310-7.

2. Robinson JC, Ex P, Panteli D. How drug prices are negotiated in Germany. New York: Commonwealth Fund, June 13, 2019 (https:// www.commonwealthfund.org/blog/2019/how -drug-prices-are-negotiated-germany).

3. Wineinger NE, Zhang Y, Topol EJ. Trends in prices of popular brand-name prescription drugs in the United States. JAMA Netw Open 2019;2(5):e194791.

4. Biosimilars in Zahlen. Berlin: Arbeitsgemeinschaft ProBiosimilars, November 14, 2019 (https://probiosimilars.de/allgemein/ marktdaten-biosimilars-september-2019/).

5. Robinson JC, Howell S, Pearson SD. Value-based pricing and patient access for specialty drugs. JAMA 2018;319:2169-70.

DOI: 10.1056/NEJMP2000341

Copyright $(\odot 2020$ Massachusetts Medical Society.

\title{
Specialty Drugs - A Distinctly American Phenomenon
}

\author{
Huseyin Naci, M.H.S., Ph.D., and Aaron S. Kesselheim, M.D., J.D., M.P.H.
}

\begin{abstract}
A ccording to a report by the Congressional Budget Office, roughly $1 \%$ of prescription drugs dispensed under Medicare Part D and Medicaid accounted for about $30 \%$ of net drug spending in each program in 2015. ${ }^{1}$ The agency found that between 2010 and 2015, net spending on these so-called specialty drugs rose from $\$ 8.7$ billion to $\$ 32.8$ billion in Medicare Part D and from $\$ 4.8$ billion to $\$ 9.9$ billion in Medicaid. Similarly, spending on specialty drugs by commercial plans nearly quadrupled between 2003 and 2014. ${ }^{2}$

The origins of the specialtydrug label can be traced back to the 1970s, when specialty pharmacies emerged in response to the need for preparation and de-
\end{abstract}

livery of new injectable and infusion products. Only a handful of drugs required such handling at the time and were called "specialty drugs." Today, various stakeholders in the pharmaceutical supply chain assign the specialty label to drugs on the basis of a combination of several unrelated factors, such as whether a drug treats a rare condition, requires special handling, or needs postmarketing risk-management plans.

But the single most common feature of specialty drugs is high cost. Indeed, the Centers for Medicare and Medicaid Services (CMS) defines specialty drugs as those with monthly costs exceeding $\$ 670$. The specialty-drug label has important consequences for patients. When Medicare Part D went into effect in 2006, CMS explicitly permitted plans to place specialty drugs on the highest cost-sharing tiers of their formularies. Today, virtually all Part D plans have a specialty tier. The maximum allowable coinsurance for drugs on such tiers is $33 \%$. A new proposed rule from CMS would allow Part D plans to implement a "preferred" specialty tier with a lower cost-sharing rate.

The economic burden of these cost-sharing requirements on patients can be substantial. Part D enrollees not receiving low-income subsidies can pay thousands of dollars out of pocket per year for a single specialty-tier drug. ${ }^{3} \mathrm{Nu}-$ merous disease-modifying therapies used for treating multiple sclerosis are considered specialty 
drugs, for example, and by one estimate, patients in standard Part D plans taking one of these drugs had average projected out-of-pocket spending of $\$ 6,894$ in $2019 .{ }^{4}$

Labeling all expensive drugs as specialty drugs and placing them on the highest cost-sharing tiers in plan formularies is an approach taken only by the U.S. health care system. By our estimate, in 2018, more than four fifths of new outpatient drugs that were covered by Medicare Part $\mathrm{D}$ during the year after they were approved by the Food and Drug Administration (FDA) were assigned to specialty tiers. Priorauthorization requirements are often implemented for drugs on specialty tiers. The specialty-drug label has become a blunt instrument for imposing financial obligations and administrative barriers on patients in response to the very high prices set for new drugs by manufacturers.

Other high-income countries undertake value-based assessments to determine whether a drug's cost is justified, given its benefits. In the United Kingdom, for example, the National Institute for Health and Care Excellence (NICE) evaluates the cost-effectiveness of a drug and makes a recommendation regarding coverage of that drug to the National Health Service (NHS). The same cost-effectiveness threshold applies to essentially all drugs (with the exception of drugs for end-of-life care and very rare conditions, for which a higher threshold is used). An expensive drug can still represent a cost-effective use of health care resources if it costs less than $£ 30,000$ (about $\$ 36,500$ ) per quality-adjusted life-year (QALY) gained. If a drug is found not to be cost-effective, the government seeks to negotiate an additional (confidential) discount with the manufacturer to push the price under the accepted threshold. Once a drug receives a positive recommendation from NICE, the NHS makes it available without cost sharing for most patients (nearly $90 \%$ of prescriptions were dispensed free of charge in 2016). If a drug receives a negative recommendation, it is not available for patients in the NHS. Coverage has been denied for less than $20 \%$ of drugs appraised in NICE's two-decade history.

Similarly, the Canadian Agency for Drugs and Technologies in Health assesses the comparative clinical- and cost-effectiveness of new drugs. This Common Drug Review process informs reimbursement decisions for Canada's federal, provincial, and territorial public drug plans, except in Quebec. Although the agency doesn't have a formal cost-effectiveness threshold, drugs that receive a positive recommendation for reimbursement generally cost less than $\$ 50,000$ Canadian (about $\$ 35,500)$ per QALY gained.

In the United States, however, expensive drugs that offer added therapeutic benefit over existing alternatives are typically placed on specialty tiers alongside other expensive drugs that don't offer such benefit. For example, sonidegib (Odomzo) was initially approved by the FDA in 2015 for treating basal-cell carcinoma. According to a comparative clinicaleffectiveness assessment conducted by the Haute Autorité de Santé, France's national health authority, sonidegib doesn't offer meaningful added therapeutic benefit over existing alternatives. Idelalisib (Zydelig) was approved in 2014 as a first-in-class treatment for certain blood cancers. It has been widely deemed to offer added therapeutic benefit over other treatments. Despite these differences, both drugs are currently placed on the specialty tiers of more than $90 \%$ of Part D plans, meaning that patients prescribed these drugs could be responsible for equivalent coinsurance rates.

We believe that the United States should abandon the nowmeaningless specialty-drug label for high-cost drugs and instead categorize therapies according to their comparative clinical- and cost-effectiveness, as is routinely done in other high-income countries, such as Canada and England. Health plans could then use valuebased categories to inform coverage and tier-placement decisions in their formularies. Comparative clinical- and cost-effectiveness analyses could be used to determine value-based price benchmarks, which would then guide decisions regarding coverage of drugs in plan formularies and reasonable prices for those drugs. More important, such analyses could inform decisions regarding copayment levels and prior-authorization requirements. Drugs that offer important added benefits over existing options and are cost-effective should not be subject to onerous cost-sharing and prior-authorization requirements. Adopting such a value-based system would improve patients' access to necessary drugs and could ultimately improve health outcomes.

The United States has a long history of devising plans to make public drug formularies more value-based that have never been fully implemented. Since the early 1970s, when the Office of Technology Assessment was created 
(it has since been disbanded), the government has made repeated attempts to incorporate comparative clinical- and cost-effectiveness analyses into formulary decisions. In another major setback for these efforts, the 2010 Affordable Care Act prohibited the Patient-Centered Outcomes Research Institute (PCORI) from conducting cost-effectiveness analyses. Although the recent Congressional reauthorization of PCORI has introduced some flexibility for the institute to "take into account" the potential economic effects of health plan benefit and formulary design, the statute establishing PCORI still forbids the use of "dollar-per-QALY" thresholds to define cost-effectiveness. In the absence of governmental guidance, independent groups such as the Institute for Clinical and Economic Review have stepped in with their own analyses of pricing structures, which have galvanized a national debate about value-based drug prices.

To reach a point where the United States can abandon the anachronistic specialty-drug label that fails to distinguish between high-cost drugs that do and those that do not offer good value for the money, public payers could learn from their counterparts in other high-income countries and formally consider clinical- and cost-effectiveness in their funding decisions. As a first step, CMS could eliminate its cost-based threshold for defining specialty drugs in Medicare Part D and instead require health plans providing prescription-drug benefits to make value-based coverage and tiering decisions. The same process could then be used for setting reimbursement levels for physician-administered drugs in Medicare Part B. We believe that patients and the health care system would benefit from evidence- based formulary designs that more accurately reflect drugs' clinical benefit and economic value.

Disclosure forms provided by the authors are available at NEJM.org.

From the Department of Health Policy, London School of Economics and Political Science, London (H.N.); and the Division of Pharmacoepidemiology and Pharmacoeconomics, Department of Medicine, Brigham and Women's Hospital, Harvard Medical School, Boston (H.N., A.S.K.).

1. Congressional Budget Office. Prices for and spending on specialty drugs in Medicare Part D and Medicaid. March 18, 2019 (https://www.cbo.gov/publication/54964).

2. Dusetzina SB. Share of specialty drugs in commercial plans nearly quadrupled, 200314. Health Aff (Millwood) 2016;35:1241-6.

3. Cubanski J, Koma W, Neuman T. The out-of-pocket cost burden for specialty drugs in Medicare Part D in 2019. San Francisco: Kaiser Family Foundation, February 1, 2019 (https://www.kff.org/medicare/issue-brief/the -out-of-pocket-cost-burden-for-specialty -drugs-in-medicare-part-d-in-2019/).

4. Hartung DM, Johnston KA, Irwin A, Markwardt S, Bourdette DN. Trends in coverage for disease-modifying therapies for multiple sclerosis in Medicare Part D. Health Aff (Millwood) 2019;38:303-12.

DOI: 10.1056/NEJMp1909513

Copyright (؟ 2020 Massachusetts Medical Society.

\section{Ensuring and Sustaining a Pandemic Workforce}

Erin P. Fraher, Ph.D., M.P.P., Patricia Pittman, Ph.D., Bianca K. Frogner, Ph.D., Joanne Spetz, Ph.D., Jean Moore, Dr.P.H., Angela J. Beck, Ph.D., M.P.H., David Armstrong, Ph.D., and Peter I. Buerhaus, Ph.D., R.N.

Current efforts to fight the Covid-19 pandemic aim to slow viral spread and increase testing, protect health care workers from infection, and obtain ventilators and other equipment to prepare for a surge of critically ill patients. But additional actions are needed to rapidly increase health workforce capacity and to replenish it when personnel are quarantined or need time off to rest or care for sick family members. It seems clear that health care delivery organizations, educators, and government leaders will all have to be willing to cut through bureaucratic barriers and adapt regulations to rapidly expand the U.S. health care workforce and sustain it for the duration of the pandemic.

As hospitals and nursing homes gear up for expected increases in critically ill patients, they should examine all opportunities to expand their workforce capacity. Where the threat of postpandemic legal consequences hampers action to expand capacity, such barriers could be removed by governors enacting emergency orders that modify or temporarily rescind medical malpractice policies that inhibit health professionals' ability to expand their scope of practice as required. Most organizations, however, will find that outdated internal policies such as workflows, task-delegation protocols, or union agreements are the main culprits in restricting the shifting of tasks and responsibilities among personnel. These restrictions can be 